\title{
TFC6 (TFIIIC subunit): a bridge between prokaryotic and eukaryotic gene regulation
}

\author{
Michèle Amouyal* \\ Interactions à Distance, Centre National de la Recherche Scientifique, Paris, France \\ *Correspondence: michele.amouyal@club.fr
}

TFIIIC is a multi-subunit protein first and best known as part of the transcriptional machinery required by RNA polymerase III for synthesis of transfer RNA (see Donze, 2012 for a recent review). Kleinschmidt et al. (2011) highlight a new aspect of this machinery, related to the sole TFIIIC, by demonstrating its capacity to directly regulate RNA polymerase II transcription - in fact, its own synthesis. The authors have dissected the role of TFIIIC and one of its subunits precisely, in vivo, providing an indepth molecular picture, which is rather unusual when dealing with eukaryotic complexity.

\section{THE MULTI-SUBUNIT TFIIIC COMPLEX AS A RECRUITING AGENT FOR THE RNA POLYMERASE III MACHINERY}

In Saccharomyces cerevisiae, the polymerase III cannot initiate transcription before a certain number of components are assembled. At genes of transfer (tDNAs), TFIIIC first binds a sequence with two conserved regions, $\mathrm{A}$ and $\mathrm{B}$. It then recruits TFIIIB, which in turn recruits the pol-III holoenzyme.

The B-box is sufficient for DNA-binding. TFIIIC is restricted to initiation and composed of six subunits. Only TFC 3 and TFC6 bind the B-box.

\section{THE TFC6 GENE}

Kleinschmidt et al. (2011) were interested in the tfc6 gene which encodes the TFC6 subunit, because the promoter contains a B-box (ETC6 site) suggesting that the gene might be auto-regulated.

Mutations in ETC6 and a highly conserved adjacent region, severely affect transcription and cell growth. Impaired cell growth is restored by the sole TFC6 complementation, indicating a simple and direct correlation with the TFC6 defect, with no other origin.

TFIIIC, TFC6 specifically, indeed binds to the tfc6 B-box and conserved region, as shown by immunoprecipitation of the promoter-bound complexes. Its binding reduces the occupancy of the promoter by the TATA binding protein of the RNA polymerase II initiating complex, presumably interfering with a pol-II upstream factor (between -120 and -40 in yeast), as TFIIIC is newly found to bind a region larger upstream (from -150 to -90 ) than the previously specified ETC6 site.

Whether by mutational analysis of the DNA or with the appropriate protein mutants, TFIIIC binding to the promoter is prevented and the level of RNA transcripts (by Northern blot analysis) is increased in the same proportion, by twofold.

Similar data with respect to colony size, are obtained when the $\mathrm{tf} 6 \mathrm{~g}$ gene is replaced by a reporter gene and when TFC6 is produced by an episomal plasmid. This effect is exclusively observed with the overexpressed TFC6, and with no other TFIIIC subunit.

\section{TFIIIC AUTO-INHIBITION: WHAT FOR?}

Auto-inhibition generally allows to strictly limit the level of a transcription factor in the cell. Pointed long ago in some model prokaryotic systems, as detailed in next section, these concentrations are determinant.

They can modify the mode of regulation. This finding allowed to unravel the contribution of non-proximal operator sequences, once thought to be cryptic, to repression of the E. coli lac operon (see Amouyal, 2006 for a review). In eukaryotes, over-expression of four key proteins is sufficient to re-program mice and human somatic cells into pluripotent stem cells like embryonic stem cells (Takahashi and Yamanaka, 2006). In Caenorhabditis elegans, it leads to terminal neuronal differentiation (Hobert, 2011).

Kleinschmidt et al. (2011) have focused on dissection of the etc6 gene and report for the first time that the level of TFC6 factor is restricted by tfc6 auto-regulation. In fact, over-expression affects cell growth. Is the production of other TFIIIC subunits also auto-regulated? TFIIIC is involved in a wide number of processes, from transcription to gene insulation and chromosomal organization (see Donze, 2012). Is its level critical for one of these processes?

This might be the case (Kleinschmidt et al., 2011). For instance, infection of human cells by the Epstein-Barr virus goes with an increase of TFIIIC concentration, which may be related to its carcinogenic potential.

\section{AS SIMPLY AS A PROKARYOTIC FACTOR}

Strikingly, it comes out from Kleinschmidt et al. (2011) work that TFC6 is much like a prokaryotic factor.

i The tDNAs and ETC sites are deprived of histones or covered with unstable histone variants, like several other insulators (Donze, 2012).

ii TFCIIIC binds strongly DNA, nearly like a prokaryotic factor, with an apparent dissociation constant of less than $10^{-10} \mathrm{M}$ at tDNAs.

iii The mechanism of tfc6 auto-inhibition resembles that of prokaryotic operons or genes. TFC6 represses its own gene by simply interfering with the binding of the pol-II complex at the promoter.

The production of a wide number of E. coli transcriptional regulators is autorepressed with help of an operator located on the promoter region or at the start of the gene. Thus, just in E. coli, over the 32 regulators reviewed by Collado-Vides et al. (1991), 19 are auto-repressed, and this number has probably expanded. The 107 listed promoters are mainly repressed through a direct interference with RNA polymerase II at the promoter, though in some instances, the repressor instead interferes with an upstream activator site or mRNA transcript elongation. The same regulator, depending upon its position with respect to the start 
of transcription, is an activator or a repressor of its own synthesis, like TFIIIC. This is the case for 6 over the 32 listed regulators.

Since these proteins are only regulatory, they do not need to be extensively produced. Thus, auto-repression limits the number of AraC molecules to 20. Alternatively, the lac repressor is maintained at the low level of 10 copies per cell thanks to a weak promoter.

Like TFIIIC, the glnG product is an auto-regulated transcription factor that contributes to gene activation. In this case, auto-repression is linked to the modulation of environmental conditions. More precisely, the glnG product (NRI) is the regulator of the system for synthesis of many enzymes required for nitrogen assimilation in enterobacteria. The heart of the nitrogen control region is the glnALG operon, endowed with three promoters, glnAp1, glnAp2, and glnLp (p1p2-glnA-p-glnLG). It comprises the glnG regulatory gene, the glnL modulator gene, and the glnA structural gene for glutamine synthetase, the sensor of ammonia availability.

In cells growing in excess nitrogen, transcription from both glnAp1 and glnLG is repressed by NRI. Under these conditions, NRI limits the synthesis of glutamine synthetase as well as its own synthesis (five molecules in the cell). Ammonia deprivation results in phosphorylation of NRI by the modulator produced by the glnL gene, activation of the glnAp 2 promoter by the phosphorylated NRI molecule and subsequent activation of a cascade of genes required under these new conditions. Activation of glnAp2 also requires a $\sigma 54$ cofactor for RNA polymerase II, used in place of the common $\sigma 70$ cofactor. Initiation of glnAp2 increases the intracellular level of glutamine synthetase and that of NRI (up to 70 molecules); This increased concentration is required for the activation of the other nitrogen regulated promoters, such as the 17 genes of the Klebsiella pneumoniae promoters of the nif regulon, or the hundred responsive genes in E. coli.

Chromosomal looping is associated with the modulation of NRI levels. It here allows to turn on the glnAp2 gene with an economy of means, since the NRI sites which were used for repression of the glnAp 1 promoter under excess nitrogen, are also used for activation of the glnAp2 promoter, but are now 100 and 130 bp upstream of the promoter. It also contributes to convert a repressor into an activator by simply changing its position with respect to the promoter.

Restricting the number of regulatory proteins is also supposed to prevent nonspecific binding and interference with other DNA-protein transactions in the cell, as prokaryotic proteins can easily bind DNA non-specifically.

Interestingly, several E. coli autorepressed repressors such as GalS or deoR, contain an internal operator within part of the gene encoding the DNA-binding region of the protein. Thus, auto-regulation might have evolved from a few common ancestor DNA-binding motifs (Roy et al., 2002).

As for NRI, auto-repression is often associated with the coupling of distant loci by chromosomal looping with help of the corresponding regulator. This is the case for repression of the already mentioned ara and deo operons. The E. coli deo operon which encodes nucleoside and deoxynucleoside catabolizing enzymes is expressed from two promoters, deoP1 and deoP2, repressed by the deoR repressor (and CytR with different inducers). It is characterized by strong promoters and high affinity DNA-repressor interactions. DNA looping allows to lock very efficiently the two promoters controlled by the same repressor, 599 bp apart, in one operation.

Another example is provided by the bacteriophage $\lambda$ (or 186) cI protein. $\lambda \mathrm{cI}$ is the repressor of the functions needed for phage replication, gene assembly, and cell lysis, thereby maintaining the lysogenic state (integration into the E. coli chromosome). Though at this stage, the virus is dormant, it is important for its survival that it can switch to the lytic state to infect other cells. However, a simple increase by twofold of the cI repressor concentration, prevents the efficient switch from dormance to virulence, requiring self-repression.

As is often the case to secure a specific process, the maintenance of lysogeny in the present case, the cell makes use of several strategies, not just one. Thus, the same molecular process, through chromosomal looping between the $\mathrm{P}_{\mathrm{L}}$ and $\mathrm{P}_{\mathrm{RM}}$ promoters, 2800 bp apart, as well as repressor oligomerization, allows (i) to stringently control the intracellular level of repressor by two means, not a single one: directly, by auto-repression of the $\mathrm{cI}$ gene from $\mathrm{P}_{\mathrm{RM}}$, indirectly by repression of the gene synthesizing the $\mathrm{N}$ protein under the control of the $\mathrm{P}_{\mathrm{L}}$ promoter, which activates repressor synthesis, (ii) to stop integrase production also controlled from the $\mathrm{P}_{\mathrm{L}}$ promoter, as it is not anymore required once the phage has been integrated, (iii) to inhibit, through $\mathrm{N}$ repression at $\mathrm{P}_{\mathrm{L}}$, several functions required for phage lysis, replication, and assembly, also controlled by this promoter.

Chromosomal looping is not consistent with the extremely high concentrations of regulator that favor the non-cooperative occupancy of all available sites (see Amouyal, 2006), as well as non-specific binding. In this context, auto-regulation would also favor chromosomal looping for the coordination and organization of gene expression when it extends over distant loci.

The coupling of distant loci within a transcriptional unit by DNA looping and regulators in E. coli, presents some common features with the organization and coordination of gene expression by the so-called gene insulators, such as CTCF (Yang and Corces, 2012). TFIIIC would even be closer than CTCF to these prokaryotic repressors, as it can also be a transcriptional repressor and as it is involved in the clustering of distant genomic loci.

Since the frontier with prokaryotes when dealing with gene expression organization, is less clear than commonly thought, one expects that the eukaryotic systems will benefit from the comparison with their prokaryotic counterparts. Conversely, the prokaryotic transcription factors presenting common features with gene insulators, might also structure the genome and perform a similar function in eukaryotic cells.

\section{REFERENCES}

Amouyal, M. (2006). Transition from DNA looping to simple binding or DNA pairing in gene regulation and replication: a matter of numbers in the cell. Gene Genomes Genomics 1, 104-111.

Collado-Vides, J., Magasanik, B., and Gralla, J. D. (1991). Control site location and transcriptional regulation in E. coli. Microbiol. Rev. 55, 371-394.

Donze, D. (2012). Extra-chromosomal functions of the RNA polymerase III complexes: TFIIIC as a potential global chromatin bookmark. Gene 493, 169-175.

Hobert, O. (2011). Maintaining a memory by transcriptional auto-regulation. Curr. Biol. 21, R146-R147.

Kleinschmidt, R. A., Leblanc, K. E., and Donze, D. (2011). Auto-regulation of an RNA polymerase II promoter by the RNA polymerase III transcription factor IIIC 
(TFIIIC) complex. Proc. Natl. Acad. Sci. U.S.A. 108, 8385-8389.

Roy, S., Sahu, A., and Adhya, A. (2002). Evolution of DNA binding motifs and operators. Gene 285, 169-173.

Takahashi, K., and Yamanaka, S. (2006). Induction of pluripotent stem cells from adult human fibroblast by defined factors. Cell 126, 663-676.
Yang,J., and Corces, V.G. (2012).Insulators, long-rangeinteractions, and genomefunction. Curr.Opin. Genet. Dev.22,1-7.

Received: 24 February 2012; accepted: 04 April 2012; published online: 24 April 2012.

Citation: Amouyal M (2012) TFC6 (TFIIIC subunit): a bridge between prokaryotic and eukaryotic gene regulation. Front. Gene. 3:64. doi: 10.3389/fgene.2012.00064
This article was submitted to Frontiers in Epigenomics, a specialty of Frontiers in Genetics.

Copyright (c) 2012 Amouyal. This is an open-access article distributed under the terms of the Creative Commons Attribution Non Commercial License, which permits non-commercial use, distribution, and reproduction in other forums, provided the original authors and source are credited. 